http://dx.doi.org/10.18778/2196-8403.2014.03

\title{
GERHARD BAUER
}

\section{In aller Büchner'schen Rauigkeit: Achtung ist ein Menschenrecht}

Prawdopodobnie najsilniejszym uczuciem, które dokuczało Büchnerowi w jego krótkim życiu był gniew z powodu pogardliwego traktowania innych ludzi. Na problem ten, będący źródłem jego głębokiego życiowego rozczarowania zwrócił uwagę podczas swoich studiów w Gießen. Problem ten napotkał również w złożonych zagadnieniach historii oraz historii literatury będących impulsem dla jego przenikliwej twórczości. Niedorzeczne błazenady, które miały uczynić znośnym życie codzienne każdego garnizonowego miasta uważał Büchner, z powodu lekceważącego traktowania bliźnich, za godne potępienia. Jego karcąca diagnoza brzmiała: Nienawiść wzmaga się. Może ona doprowadzić do aktów mordów.

Das womöglich heftigste Gefühl, das Büchner in seinem kurzen Leben zugesetzt hat, war der Zorn über die verächtliche Behandlung anderer Menschen. In seiner Studienzeit in Gießen wurde er darauf aufmerksam und wurde ziemlich bitter. In hohen Konstellationen wie der Literaturgeschichte, Anstößen seiner eindringlichen Dichtung, fand er sie wieder. Die faden Hänseleien, die das Alltagsleben einer beliebigen Garnisonstadt erträglich machen sollen, sah er durch diese schmähliche Missachtung des Nebenmenschen vergiftet. Seine strafende Diagnose: Der Groll darüber setzt sich fest. Er kann zum Mord führen.

He was a gentle and tender young man, Georg Büchner. But what enraged him really was the open or hidden contempt of other men: an affront of mankind, as he put in. He found this contempt in fellow students in Gießen. He traced it back in the turning point of the French revolution as in a silent valley of the Vosges. His Woyzeck is so much embittered by some tiny remarks of continuous disdain, so much upset of what he feels he cant grasp that he eventually murders his wife.

Gefühle: In ihnen und durch sie beglaubigen wir uns als Menschen. Das gilt eigentlich schon (um im Deutschen zu bleiben) 60 bis 100 Jahre vor dem 
Hessischen Landboten. Die ,Empfindsamkeit' fand in der Verfeinerung, also der ,Kultur' von Gefühlen ihr angemessenstes Betätigungsfeld: darin konnte sie sich nahezu straffrei ergehen. Ihre Auseinandersetzung mit dem ,allzu vernünftigen ' Rationalismus verlagerte sie am liebsten in den Kampf um die zutreffende (und zugleich schonende) sprachliche Fassung der Emotion zu gegebenen Nächsten. ${ }^{1}$ Der ,Sturm und Drang ' tobte sich einerseits aus in impulsiven Benennungen von zumeist derben Bezeichnungen, die dem ,Herzen' und seinen Launen zusetzten. Andererseits schärfte er auch das Augenmerk auf das ,Theater' der Gefühlsregungen, auf die Regie der Sprache auf dieser Bühne. Nach dem dämpfenden, um ,Gemessenheit ' bemühten Versuch einer ,Klassik' auf deutschem Boden öffneten ,die Romantiker' wieder die Schleusen, und zugleich die Ohren der Zeitgenossen, für unbeachtete Nuancen des Spiels der Emotionen. Die Bemühungen um emotionale und sprachliche Redlichkeit gegenüber dem romantischen Überschwang kamen ihrerseits nicht ohne sprachliche, also auch emotionale Entdeckungen aus. Innigkeit, Anhänglichkeit, ,concern' für einen oder mehrere andere Menschen, Emphase, Schwärmerei usw.: Die Menschheit wäre ärmer und vermutlich ruppiger im Umgang mit anderen, hätte sie nicht die lange, sich steigernde Übung in Aufmerksamkeit auf die Regungen des sprechenden Subjekts gehabt. Gefühlvoll zu sein, es im Sprechen wie im Schreiben zu beweisen, war in der Mitte des 19. Jahrhunderts in den Kreisen, die es sich leisten konnten, eine Selbstverständlichkeit, mithin also eine Pflicht. Wurde diese Standarderwartung des humanen Umgangs mit anderen enttäuscht, entstanden Fragen, Vermutungen, Argwohn. Es war nicht leicht, sie auszuräumen.

Wenn wir Büchner als Zeugen für eine grundlegende Veränderung im Gefühlshaushalt des auf sich gestellten Menschen heranziehen, wird die Situation komplizierter und schon die Fragestellung komplexer. Der Austragungsort weitet sich zum Kampfplatz zwischen einem Rest Selbstbezüglichkeit und einem Blick für die ganze soziale Umwelt. Dieser Blick hat nun Folgen auch für das Verhältnis des angesprochenen Ich zu sich selbst. Ich suche zunächst einen Begriff von dieser Veränderung zu gewinnen. Eine briefliche Selbstverteidigung des 20 Jahre jungen Studenten Georg in seinem ersten Semester an der Landesuniversität Gießen gegenüber seinen Eltern in Darmstadt scheint mir hinreichend aufschlussreich. Danach verfolge ich an dreien seiner poetischen Werke, wie die so gewonnene Haltung sich auswirkt, wenn er Dramenfiguren und den Helden seiner Novelle gestaltet. Und zwar frage ich

1 ,Nachbarn“ heißen sie bei Claudius. 
nach den wechselseitigen Beurteilungen (Schätzung oder meist Abschätzung) der Protagonisten; nach ihrem expliziten oder nur faktischen, gegebenenfalls gestischen Eingehen auf die, die als ,Geringe' gezeichnet sind; nach der Stilisierung ihres Räsonnements, mitunter ihrer Suada nach dem Prinzip des fatalen „Aristocratismus“; nach der auflockernden oder verbindenden (verbindlichen?) Rolle derjenigen Figuren, die von den Zwängen der Handlung entlastet sind: der Frauen im Danton, der einfachen Gemeindemitglieder im Lenz, des treuen Andres, des Narren, der Großmutter im Woyzeck, die zwar ohne Verbindung miteinander auftreten, aber in der geistigen Dramaturgie des Stückes etwas wie einen, Chor' bilden.

Der Brief stammt aus dem Februar 1834. Sein Original ist nicht erhalten. ${ }^{2}$ Es muss aber dem Bruder Ludwig vorgelegen haben, als er, 1850, die für ihn wichtigsten Partien daraus für seine Edition von Georgs Nachgelassenen Schriften geeignet fand. ${ }^{3}$ Der junge selbstbewusste Sohn, vor kurzem von einer schweren Krankheit (Hirnhautentzündung) genesen, an den Studienort zurückgekehrt und jetzt guten Willens zu studieren ${ }^{4}$, hat es anscheinend gegenüber seinen Verwandten und in Gießen eingeschriebenen Mitschülern an liebevollem Umgang fehlen lassen. ${ }^{5} \mathrm{Da}$ die Bekundung von Zuneigung zu den nächsten Angehörigen damals ${ }^{6}$ nicht nur erwartet wurde, sondern geradezu als Gradmesser der Zivilisiertheit z. B. eines Studenten an seinem Hochschulort galt, muss der Brief, vermutlich von der Hand des Vaters, mindestens besorgt geklungen, vielleicht auch offene Vorwürfe enthalten haben. ${ }^{7}$ Gegen diese Auslegung seines Umgangs in Gießen setzt sich der Studiosus zur Wehr. Er tut es erst defensiv, indem er sein Verhalten ins rechte Licht rückt, dann aber mit einer Retourkutsche gegen die Gießener Bekannten. Deren Umgang mit Leuten ,niederen' Standes, Bediensteten z.B., findet Georg nicht nur lieblos, sondern menschenunwürdig, gar nicht mehr zeitge-

2 Briefe sind besonders gefährdet: nicht nur wie alles Geschriebene durch den Brand von Archiven, auch schon durch Zensur durch die Obrigkeit oder durch die hütende Familie, durch Beschlagnahmung, Umzug, Verbrennen schon durch den Empfänger, z. T. auf dringendes Anraten des Absenders.

3 Dieser Ludwig war der ,Kraft-und-Stoff-Büchner', der entschiedenste Materialist der Familie, später auch Darwinist.

4 Allerdings ein Fach, das der Vater für ihn ausgesucht hat: Medizin.

5 So viel muss schriftlich oder mündlich den Eltern zugetragen worden sein.

6 In der Literaturgeschichte zählen diese Jahre zum ,Biedermeier'.

7 Er ist ebenfalls nicht erhalten, nur in seiner Tendenz aus Georgs Reaktion zu erschließen. 
mäß. Er sieht sich genötigt, die Eltern darüber aufzuklären, wie er seine Tage in Gießen verbringt. Dazu muss er sein Verhältnis zu Verwandten und Kommilitonen so darstellen, wie er es sieht. Kein Wunder also, dass sein Schreiben ziemlich grundsätzlich wird. Es stellt ein Stück praktizierte Aufklärung dar, wie sie um 1834 immerhin möglich war: „Man nennt mich einen Spötter. Es ist wahr, ich lache oft, aber ich lache nicht darüber, wie Jemand ein Mensch, sondern nur darüber, $d a \beta$ er ein Mensch ist, wofür er ohnehin nichts kann, und lache dabey über mich selbst, der ich sein Schicksal theile [...]. ${ }^{8}$

Die Anwürfe kannte er sicherlich schon aus Begegnungen mit den über ihn enttäuschten Bekannten oder Verwandten. Da hatte er nicht viel Aufhebens davon gemacht. Durch festes Auftreten diesen liebevollen Mahnern gegenüber hatte er geglaubt davonzukommen. Offensichtlich hatte er zu ihren diversen Einladungen nein gesagt und sich in seine Bude zurückgezogen. Jetzt, da seine Erziehungsberechtigten, die ihm sein Studium finanzieren, die Kritik aufgreifen, die er mündlich schon abgewiesen hat, muss er ausdrücklich und schriftlich darauf eingehen. Durch die Lebhaftigkeit seiner Argumentation verfällt er an den entscheidenden Stellen des Briefes in eine Fortsetzung seines ernsthaften Disputs mit den allzu wohlmeinenden Freunden in Gießen. Er vergisst aber an keiner Stelle, dass er seinen Eltern schreibt. Ihre Besorgnis zu zerstreuen ist, so scheint es, sein einziges Anliegen.

Die erste Hälfte des übriggebliebenen Brieffragments ist in der Argumentation defensiv gehalten, im Ton aber selbstbewusst und kämpferisch. Georg verteidigt sein Recht, sich von anderen zurückzuziehen, auch von Schulfreunden und Verwandten, wenn die ihn zu etwas Überflüssigem nötigen wollen. ${ }^{9}$ Er ficht diese Abwehrbewegung durch als sein natürliches Recht, im Ton der Allgemeinheit wie ein Kantianer und mit so viel Bemühungen um Verständnis für diejenigen, die als dumpf oder wenig ,gebildet' galten, als wollte er sogleich daran gehen, seinen Woyzeck zu schreiben. Indem er sich

$8 \quad$ Zitiert nach der Kritischen Studienausgabe der Briefe (BüchNER 1994:35f.); kursiv gesetzte Wörter: im Original meist einmal oder doppelt unterstrichen.

9 Diese Reaktion muss er gerade an der „Landesuniversität“ Gießen (wo er eben auf Bürger und Kommilitonen stieß, die einen Draht zu seinen Eltern hatten oder herstellen konnten) ziemlich deutlich ausgeprägt und trotz eindringlicher Zureden durchgehalten haben. Carl Vogt, auch ein aus Darmstadt gekommener Kommilitone, hebt trotz seiner Neugier und partieller Hochachtung in seinen Erinnerungen aus dem gleichen Winter 1833-34 Georgs ,schroffes, in sich abgeschlossenes Wesen" hervor - offenbar wirkte es abstoßend (zitiert in BÜCHNER 1999:1108) 
In aller Büchner'schen Rauigkeit: Achtung ist ein Menschenrecht

zur Wehr setzt, nimmt er sich ohne weiteres Federlesen heraus, seinerseits die Auffassung vom Menschen und seinen gesellschaftlichen Pflichten, die in diesen Vorwürfen steckt, zu zerpflücken. Seine Haltung der Selbstständigkeit und Unbekümmertheit bleibt dabei als die einzig vernünftige übrig. Niemanden „,verachten“ oder „kränken“, darauf kommt es an. Andere „meiden“ und sie sich selbst überlassen, kann gar nicht verboten werden: Jeder hat das Recht auf seine Selbstverwirklichung, auch auf einen ,Lebenswandel' nach seinem Gusto. So kommt er zu der klaren Selbstverteidigung, mit der er zugleich die anderen, „die Leute“, die das nicht einsehen wollen, dem verdienten Spott ausliefert.

Indem er die eigene Person an den Anfang der peinlichen Befragung rückt (die er in der Tat ein wenig so ausgestaltet, als stünde er vor einem Gericht), öffnet er den Blick auf das ganze zweiseitige Verhältnis. Er selbst bleibt der zum „Spötter“ Abgestempelte. Diejenigen aber, die zu einem solchen Abwehrverhalten greifen, sind dadurch ihrerseits aktiv, sie verfolgen eigene Interessen. Dadurch erscheinen sie in der zweiseitigen, personell wie charakterlich ungleichen Beziehung als die Aggressoren. Er, Georg, will nur studieren. Er will sich von der ,abscheulichen“ Stadt Gießen ${ }^{10}$ abkapseln und die dazu nötige Ruhe behalten. Sie suchen ihm überflüssige, unter seinem strengen Blick nichtswürdige, für einen eifrigen Studenten kontraproduktive Unterhaltungen und Vergnügungen aufzunötigen. Dass sie sich an seine Eltern gewandt und sie in ihr Umerziehungsprogramm zu dem damals üblichen, also ,honetten " Verhalten einbezogen haben, kann er nicht für sich thematisieren. In seiner irritierten Reaktion jedoch (sie wirkt hochfahrender, als er sonst schreibt) verrät sich, dass er hier das überschritten findet, was man sich von Kommilitonen oder Verwandten gefallen lassen muss. Ihre mündlichen Mahnungen konnte er wortlos ins Leere laufen lassen, sich einfach verhalten wie ein studierwilliger Student. Erst indem sie jene andere Instanz angerufen haben, der er Rechenschaft für seinen Lebenswandel als Student schuldet, haben sie ihm eine Antwort abgenötigt. Er verteidigt nun sein Verhalten, indem er das seiner Kritiker als ebenso vernunftwidrig wie unbedacht erscheinen lässt. Sie kümmern sich nicht um das, was sie mit ihren Einladungen und wohlmeinendem Zureden faktisch anrichten. Er als analytisch denkender Mensch bringt es auf die Formel: Sie überziehen andere, weniger Gebildete mit ihrem Spott, machen also sich selbst zum Narren. Sie haben kein Verständnis dafür, dass Georg sich im Umgang mit ihnen ,als Narr pro-

10 So entfährt es ihm kurz vor dem hier herangezogenen Brief, in einem Brief an seine Braut (zwischen dem 10. und 20.1.1834; BÜCHNER 1994:34). 
ducirt und sie dutzt“, woraus er nur schließen kann: „,ie [zu betonen: sie] sind Verächter, Spötter und Hochmüthige, weil sie die Narrheit nur außer sich suchen". Nicht dass die anderen dumm oder schlechte Psychologen wären, wirft er ihnen vor, sondern dass sie ohne Gedanken an die Wirkung für andere daherreden. Sich selbst und ihresgleichen halten sie offenbar für „Menschen“, doch anderen, die sich anders verhalten, billigen sie den gleichen Spielraum nicht zu. Sie trennen sie begrifflich wie praktisch von sich ab. Demnach wären die Sonderbarkeiten, die solche ,anderen' an sich haben, nur deren Sache, eben ihre „Narrheit“. Von der geistigen Einstellung oder dem sozialen Verhalten honetter Leute, die sich weiterbilden, die nach einer ,ordentlichen' Stelle in ihrer Gesellschaft streben - eben dieser Gesellschaft in Hessen-Darmstadt von Großherzog Ludwigs II. Gnaden - verstehen diese anderen, die ,Ungebildeten', offenbar nichts.

Es ist deren eine große Zahl, die im Besitze einer lächerlichen Aeußerlichkeit, die man Bildung, oder eines todten Krams, den man Gelehrsamkeit heißt, die große Masse ihrer Brüder ihrem verachtenden Egoismus opfern. Der Aristocratismus ist die schändlichste Verachtung des heiligen Geistes im Menschen; gegen ihn kehre ich seine eigenen Waffen; Hochmuth gegen Hochmuth, Spott gegen Spott. - Ihr würdet Euch besser bey meinem Stiefelputzer nach mir umsehn; mein Hochmuth und Verachtung Geistesarmer und Ungelehrter fände dort wohl ihr bestes Object. Ich bitte, fragt ihn einmal...

Ein Grundzug von Büchners Sozialanalyse ist damit deutlich sichtbar. Es kommt ihm nicht darauf an, den Habitus irgendwelcher unangenehmer Weggefährten zu brandmarken. Er begnügt sich damit, solche Zeitgenossen, die ihm lästig werden, die z. B. für ihre Vorhaltungen die Eltern eines harthörigen Jünglings einspannen, auf ihren Status als Mitmenschen herabzuholen. Narreteien sind zumal in einer unfreien Gesellschaft ein beliebter Gegenstand der Beobachtung, auch des Nachdenkens und der Klassifikation von anderen Menschen. Wenn aber dabei außer Acht gelassen wird (schlicht vergessen oder des Arguments wegen einfach ausgeblendet), dass die so Räsonierenden selbst Menschen sind und mit eigenen Narrheiten behaftet, dann werden solche Abgrenzungen fatal (für die anderen). Mensch zu sein ist eine dermaßen verbindende, also auch gleichstellende Eigentümlichkeit aller denkenden und redenden Wesen, dass individuelle oder kleinkollektive Superioritätsansprüche demgegenüber nichts als fadenscheinig werden. ${ }^{11}$

11 Dabei kommt es Georg nicht auf irgendeine Korrektheit der Bezeichnungen an, sondern auf die Einstellung zu dem (den) Menschen. Ein halbes Jahr später baut er in seinen beschwichtigenden Bericht über eine liebliche Nachtwanderung von Gießen nach Frankfurt ein, dass er den Weg ,theils zu Fuß, theils fahrend mit 
In aller Büchner'schen Rauigkeit: Achtung ist ein Menschenrecht

Mit dem ersten Argument hatte der gehorsame, ganz konform auf sein Studium bedachte Sohn seinen Eltern den geschuldeten Respekt erwiesen. Es hatte aber ein Manko: Es war nur logisch stimmig, nur eine erkenntnistheoretisch verfechtbare Einsicht. Andere Menschen kamen nur als die ,anderen' darin vor. Der Bezug auf den Schreiber selbst, seine Einbeziehung in das Kollektiv der irrenden und einander narrenden Menschen hatte gefehlt. Büchner baut sich den Weg zu einer solchen Demonstration am eigenen Beispiel, indem er den Spott, zu dem er sich nun einmal bekannt hat, seziert. Bei genauerer Betrachtung ist es mindestens ein doppelter Spott. Er verfügt außer über die Verachtung noch über eine Art von Spott, den „Hass“. Indem er dessen subjektives Recht entfaltet, verlässt er die spontan eingenommene Position des bloßen Verteidigers. Er zeichnet seine eigene Einstellung als Vorgriff auf eine Menschheit, die die schon vor geraumer Zeit verkündete ,égalité " praktisch, im Umgang mit anderen und womöglich allen Menschen, ernst nimmt. Jetzt sind es nicht irgendwelche, ihm herzlich gleichgültige Gießener Bekannte, die das Menschenrecht auf Gleichbehandlung, hier auf persönliche Achtung, verletzen. Sondern die soziale Rangordnung selbst, wie sie im Großherzogtum und im ganzen Land, ja im ganzen Europa (nicht nur dem damaligen) gilt, läuft auf eine fatale Ungleichheit, d.h. auch Ungleichbehandlung hinaus. Diesen durchweg unbewusst vertretenen "Aristocratismus“ greift er mit leidenschaftlichen Worten an. Er greift aus bis zum „heiligen Geist ${ }^{\text {“. }}{ }^{12}$ Anstelle seiner eigenen, hier eben umstrittenen Person bietet er den Eltern seinen Stiefelputzer als unverfänglichen Zeugen an, der in der streitigen Sache einfach der Kompetentere ist. Damit hat er nicht nur das Moment von Peinlichkeit aufgelöst, das eine Selbstrechtfertigung eines ,fast erwachsenen" Menschen gegenüber seinen Eltern an sich hat. Er hat überhaupt das Verhältnis des Gegenüberstehens durchstoßen. Sich selbst muss er zwar seinen Ernährern gegenüber in ein richtigeres Licht setzen, aber auf sich, auf seine noch so einleuchtende Problemlösung soll es gar nicht ankommen.

Postillonen und sonstigem Gesindel“" zurückgelegt habe (3.8.1834 an seine Familie; BÜCHNER 1994:46).

12 Vermutlich verbindet er als erklärter Atheist mit dieser gewaltigen Vokabel kaum irgendwelche religiösen Gefühle oder Kindheitserinnerungen. Ihm geht es um die Aufdeckung des Heiligen Geistes „,im Menschen“: in einem jeden, unabhängig von seiner Bildungsstufe. Deshalb hütet er sich auch, seine Eltern belehren zu wollen oder sich als Musterknaben hinzustellen. Sie sollen ihre eigene Aufmerksamkeit auf die derzeitige Erfüllung oder Verletzung des kaum noch streitig zu machenden Menschenrechts entwickeln. 
Wenn seine Eltern (sein Vater vor allem) sich um solche Fragen kümmern wie den angeblichen „Hochmuth“ ihres Filius, dann sollen sie sich bei einem Menschen erkundigen, der ihn gut genug kennt und demgegenüber er offenbar nie den ,Herrn“ gespielt hat. (Er konnte sicher sein, dass sie von diesem ,Angebot' keinen Gebrauch machen würden.)

Sein Beispiel ist freilich nur bei einer sehr generösen, getreulich ,mitgehenden ' Lesart annähernd überzeugend. Seine Diktion verrät ebenso wie die Argumentation (die beiläufig wirken soll, doch ziemlich aufdringlich klingt), dass er in dem gesellschaftlichen Neuland, für das er sich so engagiert, noch nicht recht angekommen ist. Den Mann, der ihm in jedem Bedarfsfall sein Schuhwerk säubert, nennt er (wie seine soziale Klasse überhaupt) „mein Stiefelputzer" - als ob der dazu geboren wäre, als ob die ganze (wenig prosperierende) Wirtschaft des Großherzogtums ihm keine alternative Beschäftigung bieten könnte. Die Bedienung gerade in diesem heiklen Punkt, in dem Schmutz und Sauberkeit strikt nach der Standesgrenze verteilt sind, sowie die Zuordnung wie ein persönliches Eigentum akzeptiert er, als wären sie von Natur gegeben. Sein Interesse, im Gespräch glimpflich davonzukommen, verteidigt er wie ein ausgemachter Egoist. Gut ist, was ihm am wenigsten Skrupel bereitet: „Alles, was existirt, bey seinem Namen zu nennen ${ }^{13}$ und dem, was mir unangenehm ist, aus dem Wege zu gehen“. Es kommt ihm aber nicht auf die Schönheit oder auch nur auf die Konsistenz seines Arguments an; die Diktion nimmt er ohnehin auf, wie sie sich ihm aufdrängt. In aller Fehlerhaftigkeit oder Willkür gelingt es ihm, zwei Punkte des erforderlichen Umdenkens klar herauszubringen. Er achtet den Mann, der ihm die Stiefel putzt, wie seinesgleichen, und er erteilt ihm das Wort.

Auch so bleibt es ein höchst eigenwilliger, entschieden subjektbetonter Materialismus, dem der junge Revolutionär sich hier verschrieben hat. Einen Monat bevor er mit Gießener Freunden (nach dem Vorbild aus Darmstadt) eine „Gesellschaft für Menschenrechte“ gründet und seine Vorstellungen für eine Agitation unter den vielfältig bedrückten Bauern des Großherzogtums zu Papier bringt (den späteren Hessischen Landboten), behandelt er soziale Verhältnisse im persönlichen Bereich so, als ob sie als materielle Fakten gar nicht zählten. Als ob das Entscheidende an ihnen die innere Einstellung wäre, die sich im Achtungserweis beglaubigen soll. Wichtiger als die soziale Revolution und eine erwünschte spätere Gleichstellung ist ihm die Verquickung

13 Hier begnügt er sich mit dem schlichten „Nennen”, als wollte er beim alten Nominalismus hospitieren. 
subjektiver und objektiver Faktoren in jedem sozialen Verhältnis. Auf sie konzentriert er sich und richtet seine Emphase auf ihre praktische Verwirklichung. ${ }^{14}$ In der prekären Situation, dass seine Eltern ihm vorhalten, was die Gießener Bekannten/Verwandten ihnen zugetragen haben, unter dem Druck der Aufklärungspflicht sind diese Passagen offenbar das Maximum, was der so ins Gebet genommene Sohn leisten (aus sich leisten) konnte. Der Passus, den sein Bruder später auswählt, schließt mit dem Selbstbekenntnis oder der Selbstverpflichtung: „Ich hoffe noch immer, daß ich leidenden, gedrückten Gestalten mehr mitleidige Blicke zugeworfen, als kalten, vornehmen Herzen bittere Worte gesagt habe.“

Damit ist der Skopus des Briefeschreibens klar überschritten: auch auf den einen Zeugen für sein Verhalten kommt es nicht mehr an. Hier spricht der 20jährige Büchner sein literarisches Programm aus. Er konnte nicht wissen, dass er nur noch drei Jahre Zeit für die Ausführung hatte. Die Passagen seines ersten Dramas, in denen Danton und einige der Seinen auch mal von sich sprechen, die vom Mitgefühl dirigierte Erzählung Lenz, die mehrfach neu ansetzenden Entwürfe zu einem Woyzeck-Drama, in dem die Zentralfigur am Sprechen vielfältig gehindert wird und gerade dadurch sich als Mensch ausdrückt, lösen manches von diesem weit gespannten Programm ein. Sein Lachen vergeht ihm nicht dabei, es wird nur noch beträchtlich bitterer. ${ }^{15}$ Das Leiden, das in diesem Brief so ernst genommen wird ${ }^{16}$, gewinnt dort seine ausschlaggebende Rolle: in der Philosophie und Spekulation, im Austausch von Emotionen, im Grübeln über sich selbst wie in der Neigung zu einer anderen Person; wenn einer die Kanzel besteigt, dann wie selbstverständlich auch in der Predigt. Bei Adorno, der manche Einsichten in seine geistfeindliche Gegenwart Büchner verdankt, ist „der Schmerz" aus der Philosophie

14 Auch sonst klaffen manchmal bei ihm, z. B. gegenüber ihm bekannten Menschen, der Stand seiner gelehrten Kenntnisse und sein Umgang mit Personen auseinander. Von seinem Onkel Georg muss er sich vorhalten lassen, dass er „mit viel Kenntnißen“ doch „als ein Schußel auf der Welt herum gehe“ (24.3.1834 aus Darmstadt; BüCHNER 1994:41).

15 Politische Bewegungen der Familie in Darmstadt zu melden und sie zugleich als „Komödie“ oder „Satyre“ zu relativieren, war die Schreibstrategie des politischen Anfängers, des Studenten in seinen ersten Semestern gewesen; s. die Briefe ,nach dem 4. Dezember 1831“, Dezember 1832, ,um den 6.4.1833“, 12. Oder 19.11.1833 und 9.12.1833, alle an seine Familie in Darmstadt (BÜCHNER 1994: 7f., 18, 20f., 31, 32f.).

16 Selbst „das Geistige“ zeigt sich vor allem darin, dass etwas wehtut, und zwar einem der „Geringen“. 
nicht auszutreiben: ein „Datum“, an dem die Strategien des darüber empörten Denkens sich eher blamieren, als dass sie es irgendwie beheben. Cornel West, der auch als Philosoph noch Prediger bleibt, sieht die Kernaufgabe der Philosophie in der „Arbeit an der Sichtbarmachung des Leidens“.

Gleichwohl ist es beim Übergang vom persönlichen Zeugnis zu dem der literarischen Werke unerlässlich, die Eigengesetzlichkeit der Literatur zu achten. Die Figuren, die der junge Autor auswählt, um Probleme anzupacken, mit denen er in seinem Privatleben z.B. als Student nicht fertig geworden ist, werden dadurch nicht zum Sprachrohr seiner persönlichen Ansichten. Sie wollen und sollen weder schon gefestigte Überzeugungen von ihm noch seine ungelösten, vielleicht für ihn gar nicht lösbaren Fragen weitertreiben. Auch wenn sie manche Lieblingsvorstellungen von ihm aufnehmen, bleiben sie ,fremde' Personen. Er kann sie sich oder auch ,uns' nahe rücken, diesem imaginären Kollektiv, das er durch sein Schreiben und das die gegenwärtig Nachlebenden durch eine Aufführung seiner Dramen konstituieren. Er bleibt jedoch ein anderer als die liebenswürdige Lucile, als der spaßig-ernsthafte Valerio oder der geplagte Woyzeck. Wo er zu intensiver innerer Teilnahme einlädt, baut er immer auch kleinere oder größere Sperren gegen die schiere Identifikation ein. Indem er mit solchen Spielfiguren historische und soziale Bereiche erkundet, die ihm in seinem Lebenslauf erspart geblieben sind, gewinnen sie ihre eigene Stimmigkeit und Stärke. Ihre Lebensumstände sowie ihre persönlichen Strategien, sich in diesen Umständen zu behaupten, plagen sie stärker als diejenigen Überzeugungen und Wünsche, die sie vielleicht mit ihrem sozial weitsichtigen Autor gemeinsam haben.

Dantons Tod (BÜCHNER 1992:11-90) ist ein hartnäckiges Drama über das Leiden und seine angebliche Unvermeidlichkeit, und zwar bis zur Folter, die auf der Ebene der stark gewählten Worte immer wieder durchscheint. In der Frage, welches Recht es für gedankenlos aggressive Verhaltensweisen gegenüber ,Niederen' geben könnte, ist das Drama als Ganzes radikaler als seine handelnden Personen. Man muss auf das hören, was sie und wie sie reden; man darf sich nicht ablenken lassen auf die meist kurzschlüssigen Ziele, die sie verfolgen. Der Titelheld und, nicht zu vergessen, die übrigen Revolutionshelden und -mitläufer streiten sich vor allem über die Gewalt, die ihnen so zusetzt, weil sie sie bei ihrem Hauptanliegen selbst nicht entbehren können. Danton selbst scheint die Notwendigkeit der Revolution ebenso wie ihre Beendigung zu bejahen - darf man aber die eine wie die andere Zustimmung gleichermaßen ihm als Person zuschreiben? 
In aller Büchner'schen Rauigkeit: Achtung ist ein Menschenrecht

Wie nehmen die Helden dieser Revolution Personen, niederen' Standes oder geringer Relevanz wahr, wie behandeln sie sie? Der junge Autor lässt in seinem Drama Mitspieler auftreten, denen Danton hochmütig vorkommt. Billaud-Varennes findet Dantons Auftreten von einer ,feinen Aristocratie der Menschenverachtung“ gezeichnet. Seinen Blick empfindet er wie „von oben herunter“. „Das Volk“ täte gut, ihn sich nicht länger gefallen zu lassen (III/6). ${ }^{17}$ Von einem solchen Mitkämpfer angeschwärzt zu werden schadet zwar der Reputation, bringt aber einen Mann wie Danton noch nicht in den Kerker. Zwei „Bürger“ aus einem „Volkshaufen“ kommen wenig später (III/10) in einem abenteuerlichen Argumentationsgang darauf, der so empörend zur Schau gestellte Luxus des einstmals „armen“ Advokaten Danton könne nur aus der Bestechung durch die abgesetzten adligen Größen stammen. Damit ist sein Todesurteil besiegelt, erst in ihren Worten, dann in der politischen Wirklichkeit. Dieser aufreizende Reichtum und vor allem die Nonchalance, mit der Danton und seine Anhänger ihn genießen, ist in der Tat skandalös. Die Nutznießer behandeln ihn aber nicht als Beweis für Dantons Käuflichkeit, sondern als Provokation, die das Denken weitebringen soll. Danton hat keine Skrupel, die ihm zugefallenen Güter zu seinem Genuss zu nutzen. Aber er sucht nirgends der Verantwortung gerecht zu werden, die er dadurch auf sich gezogen hat. Er macht Ansätze, darüber zu grübeln, und scherzt dann wieder das Anstößige hinweg. Das Lachen wird im ganzen Stück der Modus, in dem das in sich Widersprüchliche, vielleicht Unauflösbare oder direkt Absurde dieser Stegreifrevolution kenntlich gemacht und anerkannt wird. ${ }^{18}$ Der Vorwurf der Käuflichkeit gilt als plump, nur äußerlich. Fataler ist, dass die Selbstbestimmung des Handelns nur in Worten in aller Striktheit (fast wie bei Kant) postuliert wird, dass sie aber vor dem Automatismus dieser Revolution versagt.

Im einleitenden Salongespräch, gewissermaßen im Hauptquartier der Dantonisten, ist es nicht der Titelheld, sondern Hérault de Séchelles ${ }^{19}$, der in der Form des Postulats vertritt, was der Verfasser des Dramas ein Jahr zuvor

17 Billaud war selbst durch die Revolution sozial aufgestiegen: zum Mitglied des ,Wohlfahrtsausschusses “ und Präsidenten des Konvents. Er spricht mit der Drastik des siegreichen Revolutionärs; er war auch an den entscheidenden Events dieser Revolution aktiv beteiligt.

18 Auch Dantons Widersacher streiten darum, ob die Revolution die Guillotine zum Lachen bringen dürfe - wo bliebe dann der Respekt der Leute? (III/6)

19 Aus altem Adel, aber seit Beginn der Revolution deren kräftiger und erfolgreicher Mitstreiter. 
gegenüber seinen Eltern verfochten hatte (I/1). „Jeder muß sich geltend machen und seine Natur durchsetzen können. Er mag nun vernünftig oder unvernünftig, gebildet oder ungebildet, gut oder böse sein, das geht den Staat nichts an. ${ }^{\text {‘20 }}$ Über die unvermeidliche Narrheit der Menschen eben als Menschen hat der junge Autor gegenüber dem ,Einfall ' im Brief an die Eltern vor einem Jahr weiter nachgedacht. So lässt er hier sein auffälliges Sprachrohr hinzufügen: „Wir Alle sind Narren es hat Keiner das Recht einem Andern seine eigentümliche Narrheit aufzudrängen.“ Danton ist hier abgelenkt, er philosophiert gerade mit oder für Julie. Aber noch im gleichen Akt (I/6) macht er daraus sein entscheidendes und deshalb auch das empfindlichste Argument gegenüber Robespierre: Er empört sich gegen dessen immer gleichbleibende „Rechtschaffenheit“. Jahrelang mit der gleichen „Moralphysiognomie“ herumzulaufen, bloß um des „Vergnügens“ willen, „Andre schlechter zu finden“" als sich selbst, kommt ihm so verkrampft wie unökonomisch vor. Dass dieser einstige Freund, jetzt der Führer der Revolution, sich wehrt, wenn andere ihm auf seine peinlich weiße Weste spucken, das kann er verstehen - ,aber was geht es dich an, so lang sie dich in Ruhe lassen?" Die gleiche Gegenüberstellung wie in jenem Brief bekommt hier einen politischen Akzent. Die Freiheit, sich um das Benehmen von anderen zu „,kümmern“ oder sie „,in Ruhe [zu] lassen“, hat sich im Laufe der ständig gefährdeten Revolution verschärft zur Alternative zwischen GuillotinierenLassen und Am-Leben-Lassen. Die Revolution als Revolution weiterführen heißt, alle sonstigen Ziele der ausgerufenen „,Tugend“ = Einseitigkeit = Härte aufzuopfern, also auch alle Mitspieler. „Die Lava der Revolution fließt“, sagt Collot „mit Pathos“ zu dem Regisseur und Instrukteur dieses Schreckensregimes St. Just. ${ }^{21}$ Gegen so viel habituell gewordene Perversion hilft weder der Leichtsinn dieser selbsternannten „Römer“ (II/1) noch die Suche nach einem anderen Verhältnis zwischen Literatur (oder Theater) und Wirklichkeit (II/3, IV/3), auch nicht die Aufbauschung zu noch so innig-melancholischen

20 ThOMAS Michael MAYeR (1988) hat noch einen Satz davon entziffert: „Dieser Satz muß Grundsatz des neuen G ... [Gesetzes?] werden." Die Vorstellungen, wie die „Republik“ die „Revolution“ ablösen könnte: „Recht“ statt „Pflicht“, „Wohlbefinden“ statt „Tugend“, „Notwehr“ statt „Strafe“, würden damit kräftig unterstrichen. Vielleicht zu eindeutig, zu ,laut" für den auf Dezenz und historische Plausibilität setzenden Dramenautor?

21 Dumas, einer der Präsidenten des Revolutionstribunals, gibt im Schwung der Begeisterung und der Denunziation gleich seine Frau preis. Er beruft sich dabei auf Brutus, der ebenfalls ,sein Liebstes dem Vaterlande“ geopfert habe (IV/2). 
Protestformeln gegen die Aufopferung real empfindlicher Nerven für mehr oder weniger gelungene ästhetische Effekte (IV/5). „Die Welt ist das Chaos“, resümiert Danton. „Das Nichts ist der zu gebärende Weltgott.“ (IV/5) In St. Justs ebenso kalter wie hingebungsvoller Begeisterung für „die Natur“, die in ruhigen Zeiten wie in Revolutionen ihren „Gesetzen“ folgt und dabei „Millionen“ von Menschenleben hinrafft, sind „Leichen“, die der „Strom der Revolution“ bei jeder Krümmung ,ausstößt“, gewissermaßen Kosten des Fortschritts. Er ist nicht nur aufrichtiger als die anderen „Banditen der Revolution ${ }^{، 22}$ mit ihren durchweg egoistischen Motiven. Er sagt, dass auch „einige hundert Leichen“ ihn und die Seinen nicht hindern sollen, ihrem „Satz" noch „einige Schlüsse hinzuzufügen“ (II/7). ${ }^{23}$ Barrère de Vieuxsec, der sich ${ }^{24}$ einiges herausnehmen kann, sagt sein Amen zu der blutdürstigen Haltung wie der Mischung von sprachlicher Prägnanz und Henkerskunst: „Ja, geh St. Just und spinne deine Perioden, worin jedes Komma ein Säbelhieb und jeder Punkt ein abgeschlagner Kopf ist.“ (III/6) Kein Wunder, dass auf der „Gasse“, unter den „Bürgern“ des Volkes, die gleiche Logik die drastischsten Formen annimmt: „Totgeschlagen, wer lesen und schreiben kann!“, „Totgeschlagen, wer auswärts geht!“ (I/2) Die Verunsicherten um Danton wollen diese Logik nicht länger bedienen. Noch in ihre Absageformeln nehmen sie den Automatismus der triumphierenden Rücksichtslosigkeit auf. „Wie lange sollen wir Algebraisten im Fleisch beim Suchen nach dem unbekannten, ewig verweigerten x unsere Rechnungen mit zerfetzten Gliedern schreiben?" (Camille, $\mathrm{II} / 1)$

„Die Revolution“ scheint auch diejenigen, die ihr Einhalt gebieten wollen und vielleicht könnten, in ihren kontraproduktiven Automatismus zu verstricken. Robespierre kann nach seinem aufwühlenden Gespräch mit Danton nicht einfach weitermachen wie bisher. Er kann aber auch die Revolution, die er maßgeblich mit in Gang gesetzt hat, nicht stoppen. Selbst dem zu allem

22 Auf die Einwände seiner Familie gegen die furchtbaren Ausdrücke dieser Revolutionsgrößen antwortet Georg in einem späteren Brief (28.7.1835; BÜCHNER 1994:74), sichtlich ohne Bedauern, er habe diese „Banditen der Revolution“ nicht gut zu „Tugendhelden“ umstilisieren können.

23 Die durchgehende Verschwisterung von Rhetorik und Terreur bleibt sonst meist unausgesprochen, nur fahrlässig angespielt, doch im Unterbewusstsein präsent. Hier wird sie vom sachverständigsten und Veranstalter einmal mit nonchalanter Brutalität in Worte gefasst.

24 Als ehemaliger Anwalt am Parlamentsgericht von Toulouse und Abgeordneter bei den Generalständen. 
entschlossenen St. Just gelingt es nicht, die aufgeworfenen Zweifel und Selbstzweifel zum früheren Gleichmut zurückzubiegen. Robespierre sieht sich nun nicht mehr als Urheber dieser Revolution, sondern als einen Vollzugsbeamten. An ihre Heilsamkeit kann er nicht mehr glauben, kann aber auch nicht aussteigen. Dantons Vergleich zwischen ihm und Christus, verschärft durch die boshafte Formel „Polizeisoldat des Himmels“, hat in ihm eine Gedankenverkettung freigesetzt, aus der er sich, schließlich allein, nicht lösen kann. Das Bild vom „Blutmessias, der opfert und nicht geopfert wird“ muss er auf sich sitzen lassen. Er findet seine Position, und jetzt seine Aufgabe, noch fataler als die des damaligen ,Erlösers“: „Er hatte die Wollust des Schmerzes und ich habe die Qual des Henkers." Das sagt er, als die Hinrichtung der „Gemäßigten“ noch nicht beschlossen ist (I/6). In ihm, auf den (noch) so viel ankommt, scheint sie schon festzustehen. Diese Einsicht hilft ihm aber weder weiter noch zurück. Er bemitleidet sich so intensiv in seiner Rolle als letzter Ausführender, dass er sich den Weg zurück zum entscheidenden Urheber des Urteilsspruchs selbst verbaut.

Lucile, weit weniger verstrickt und ganz machtlos, aber stärker betroffen, malt sich einen anderen Ausweg aus. Wie ein Kind sucht sie ihn sogleich, jedoch ohne Ausdauer, in die Tat umzusetzen. „Alles stockt“, empfindet sie. Sie will bewirken, dass wirklich alles stockt. Sie setzt sich auf den, verhüllt ihre Augen und schreit. „Nach einer Pause“ erhebt sie sich aber und konstatiert, dass sich nichts geändert hat. „Wir müssen’s wohl leiden“ (IV/8). Eine andere Konsequenz sucht sie in der letzten Szene (IV/9) mit einer unvermuteten Sprachgeste, die in der wendungsreichen Auslegung sowohl als Selbstdenunziation wie als „Majestät des Absurden“ (Celan) gewürdigt wurde. Ohne jeden Anlass, sicher ohne Anwandlung des inzwischen verpönten Royalismus ruft sie laut ${ }^{25}$ : „Es lebe der König!“ Die hier wie überall patroullierenden „Bürger“ nehmen sie daraufhin fest, „Im Namen der Republik“, und führen sie ab. Das Schicksal des historischen Vorbilds von Lucile ${ }^{26}$ kann der Autor damit nicht mehr ändern. Aber als Protest gegen eine dermaßen humorlose, schematische Beendigung eines Menschenlebens spielt der erdachte ${ }^{27}$ Schluss Büchners noch einmal zeichenhaft, weiblich, impulsiv die Liebe zum Leben

25 In der Regieanweisung hat Büchner vorgesehen: „sinnend und wie einen Entschluß fassend, plötzlich“" (BÜCHNER 1992:584).

26 Camilles Frau wurde wegen illegaler Unterstützung der Gefangenen eine Woche nach Camille ebenfalls guillotiniert.

27 Immerhin stützt er sich auch auf einige überlieferte Vorgänge unter Frauen und Witwen von Revolutionären. 
In aller Büchner'schen Rauigkeit: Achtung ist ein Menschenrecht

aus. Es musste etwas Unerwartetes sein, etwas historisch Unpassendes, das in jener Zeit schlechterdings verfemt war, womit die ebenso sensible wie energische Lucile ihr Plädoyer für eine , andere' Art, Geschichte zu machen, unterstreichen konnte.

Lucile, der Liebling der Interpreten, verfällt auf diesen Ausweg, im Namen des Lebens und mit dem Ausruf ,es lebe“ ihr Leben zu beenden, wie auf einen plötzlichen ,Einfall‘. Dantons Gattin Julie ${ }^{28}$ „geht“ stiller, ohne Demonstration, nur im suggestiven Einklang mit ihrem Mann, der nicht mehr zu retten ist (IV/1 und IV/6). An der Grisette Marion ${ }^{29}$ gestaltet Büchner etwas wie die Verherrlichung (und Verewigung) der Prostitution. Für sie ist alles eins, wie sie selbst ,,immer nur Eins“ ist: „Ein ununterbrochenes Sehnen und Fassen, eine Glut, ein Strom“ (I/5). Dazu leiht der Autor dieser außergewöhnlichen Figur so schmelzende, eingängige Worte (,wer am Meisten genießt, betet am Meisten"), dass die meisten Interpreten auf die grobe Vereindeutigung der weiblichen Existenz gar nicht eingehen, nur die völlig entlastete Ekstase des Gefühls und meistens noch die Schönheit der Sprache preisen.

Büchners erstes Drama ist in seiner Penetranz wie seiner Sprunghaftigkeit ein starkes Plädoyer für das Menschenrecht Achtung vor einer jeden menschlichen Kreatur. Fast alle sprechenden und handelnden Figuren sind jedoch zu stark fixiert an die Ziele, die sie verfolgen. Oder sie sind zu perplex über die erlebten Brüche und Lücken in der Konsequenz ihres (und allen) Handelns, als dass sie sich zu einem überzeugenden Verfechter dieser Einsicht eigneten. Gerade durch dieses andeutungsweise Thematisieren, ohne dass sie es propagieren $^{30}$, wird das Desiderat zu einem wirklichen Desiderat. Es ist wünschenswerter als alle im Stück verfolgten Teilziele, ist aber auch schwieriger zu erlangen. Kein Geniestreich, zumal nicht auf dem Theater, kann es einfach bescheren. Nur durch lange Mühen der Reflexion, der Selbstkritik und Selbständerung, der öffentlichen Aufklärung, der Revolutionierung der sozialen Verhältnisse könnte das Volk sich ihm allmählich nähern.

28 Ihr historisches Vorbild lebte noch jahrzehntelang weiter.

29 Eine freie Zutat des Autors zu seinem sonst überwiegend historischen Material.

30 Überdies in einer historischen Konstellation, die bereits 40 Jahre zurücklag und deren Ausweg Revolution viel skeptischer betrachtet wurde, selbst von diesem Freund der Revolution, der deshalb seine Flucht aus seinem Land vorbereiten musste. 
Im Danton hat Büchner das Dialogische der Handlung wie der Gestaltung, also auch der Äußerungen stark unterstrichen. ${ }^{31}$ In dem knappen, jedoch liebevoll und höchst aufmerksam gestalteten Prosatext über den unglücklichen Lenz bei seinem letzten freien Besuch ${ }^{32}$ geht es um ein ganz anderes Projekt. Sein Verhältnis zur Natur, zur Tradition ${ }^{33}$, zur Bildung, zur Religion steht im Vordergrund. Er arbeitet an einer Neudeutung der ganzen Welt um ihn. Die auch hier bedeutungsvoll eingebauten Dialoge sind durchweg Kontroversen. Nur wenn einer einmal mit Autorität sprechen darf (Lenz auf der Kanzel) und die Gemeinde wie üblich nur zuhört, kommt ein friedvoller Gleichklang zustande. Die dafür nötige Willkür wird aber deutlich herausgestrichen: Der Prediger ist eigentlich oder wird im Laufe der Handlung Atheist. Er knüpft nur gern an eine gern erinnerte Phase seiner Ausbildung und seines einstigen Glaubens an. Er gibt sich Mühe, „einfach“ mit den Leuten zu sprechen. Er hat die Genugtuung, dass sie alle mit ihm ,leiden", und bildet die Hoffnung aus, „diese dumpfen Leiden gen Himmel leiten“ zu können (BÜCHNER 1992:230-232). ${ }^{34}$ Die psychischen Kosten aber, mit denen er dieses begütigende Zureden bezahlt, werden anschließend mit großer Eindringlichkeit dargestellt. „Musik“ und „Schmerz“ in ihm erschüttern ihn so, dass er die Einsamkeit seines Zimmers suchen muss. Hier „krümmt“ er sich erst recht, „es war ihm als müsse er sich auflösen“. Sein Mitleid kreist jetzt um ihn selbst. Er weint über sich; nur so findet er schließlich in den Schlaf. Im Kunstgespräch mit Kaufmann setzt er der gerade grassierenden ,idealisti-

31 Dantons ,Fraktion' bildet eine Art ,Chor', der die verwegenen bis desolaten Situationsbestimmungen ihres Helden ergänzt, abwandelt oder weitertreibt, wo es not tut auch vorbereitet. Seine junge Frau und eine Reihe von Grisetten ergänzen das Bild eines dialogisch lebenden und denkenden Teilnehmers jener aufregenden Geschichtsepoche. Die Leistung und der Trug der Sprache wird durch mehrere ,Echos“ von Reden, durch Übernahme von Gedanken oder Kadenzen aus der Rede des einen in die eines Freundes oder Kontrahenten sowie durch mutwillig artikulierte Grübeleien über dieses Hin und Her hervorgehoben. „Ich werde, du wirst, er wird“, antwortet Danton auf Camilles Auftrag. „Du wirst den Angriff im Konvent machen.“( $\mathrm{I} / 1$ )

Bei Pfarrer Oberlin und seiner Gemeinde in einem entlegenen Tal der Vogesen. Der anerkannt hohen ebenso wie der ,geringen”, volkstümlichen.

34 Die Gemeinde bleibt zwar in der ganzen Novelle passiv, zumeist stumm. Aber in ihrer bloßen, öfter erwähnten Existenz, als Adressat des rührigen Pfarrers, der sie alle, jeden nach seinen Fähigkeiten, unermüdlich zur Tätigkeit ermuntert, wie als Gemeinde zu Füßen dieses liebevoll zugewandten Hilfspfarrers bildet sie, dramatisch gesprochen, eine Art von, Chor'. 
schen Kunst“ [sic] entgegen: sich ,in das Leben der Geringsten“ versenken. In seinen beiden Dramen Hofmeister und Soldaten habe er es ,versucht“. Es darf einem keiner zu gering, keiner zu häßlich sein.“ (BÜCHNER 1992:234f.)

So denkt, fühlt und macht sich der in seinem Inneren bedrohte Poet die Welt zurecht. Sie muss schlicht und gut verständlich sein, möglichst irgendwie anheimelnd. Die Menschen müssen keineswegs schön sein, aber die lebenden Bilder, die sie mit ihren Interaktionen abgeben, bilden seinem Bericht nach eine „schöne Gruppe“. Sie übertreffen noch „die schönsten, innigsten Bilder der altdeutschen Schule“. Da werden die Haare, die das eine Mädchen dem anderen zu binden hilft, zum „goldnen“ Haar; das „bleiche Gesicht“ und die „schwarze Tracht“ kontrastieren stilvoll dazu. Kaum hat der Beobachter das Gesehene auf eine solche harmonische Formel gebracht, will er ihm auch Dauer verleihen. Dazu greift er zu einem fatalen Mittel: „Man möchte manchmal ein Medusenhaupt sein, um so eine Gruppe in Stein verwandeln“ und es den Leuten zeigen zu können (BÜCHNER 1992:234). Sein Inneres ist dermaßen erfüllt von dem lieblichen und würdevollen Anblick, dass er sich nicht damit begnügen kann, ihn $\mathrm{zu}$ einem tragenden Baustein seines Kunstbekenntnisses zu machen. Er will ihn auch draußen zur angelegentlichen Belehrung für jedermann, jeden aufmerksamen Passanten also, verewigen. Aus dieser Stimmung und Intention stammt sein schärfstes Urteil in diesem Wort-Streit um die richtigste künstlerische Wiedergabe der in Gestalten verkörperten Natur. „Dieser Idealismus ist die schmählichste Verachtung der menschlichen Natur." (BÜCHNER 1992:234) Hier greift die Figur Lenz zu so starken Worten wie in jenem Brief vom Februar 1834 der junge Büchner persönlich, um den „Aristocratismus“ des Verhaltens in seiner vorsätzlichen oder achtlosen Überheblichkeit über die „Geringen“" zu verurteilen. Er spricht so definitiv, als wäre der Jüngste Tag angesagt.

In einer Hinsicht wird hier die Intention jenes zurückliegenden Briefes konsequent weiterentwickelt. Es geht nicht mehr um die Fürsprache eines Gebildeten und Gesicherten für einen solchen „Geringen“ ${ }^{35}$ Hier bekommt einer der Geringen selbst das Wort - und zwar in einem interessanten Fall, weil dieser Lenz immerhin schreiben kann. Eine seiner „Komödien“ ist zu seinem Erstaunen dem Pfarrer Oberlin bekannt. Einen Stiefelputzer wirklich zum Sprechen zu bringen wäre schwierig; das bleibt eine Phantasie, die aus Büchners Brief über den Brief hinausreicht. Ein empfindsamer, leidenschaftlicher,

35 Außer wenn man schon die Bemühung des erst halb arrivierten Autors um den „armen Lenz“ als einen solchen Akt der Stellvertretung gelten lässt. 
momentan erregter junger Poet aber lässt sich nicht erst bitten. Er bricht von selbst in Klagen, Anklagen, Tränen, Selbstbeschuldigungen und Selbstrechtfertigungen aus. „Laßt mich doch in Ruhe!“ sagt er ohne wörtliche Veranlassung zu Kaufmann wie zu seinem Vater, dessen Interessen Kaufmann hier zu vertreten sucht. (BÜCHNER 1992:236) „In einzelnen Augenblicken“ fühlt er zwar (,tief“), „wie er sich Alles nur zurechtmache“ (BÜCHNER 1992:237). Meistens aber ist er so erfüllt von dem, was ihm auf seinen ziellosen Wanderungen zustößt und was er sich daraus ,zurecht macht“, dass er wie hingegeben reagiert, meist auf einer hohen Stufe der Erregung. Nach der fehlgeschlagenen Auferweckung eines toten Mädchens ist es ihm, ,als könne er eine ungeheure Faust hinauf in den Himmel ballen und Gott herbei reißen“, „als könnte er die Welt mit den Zähnen zermalmen und sie dem Schöpfer ins Gesicht speien“ (BÜCHNER 1992:242). Er will eigentlich nichts als „Ruhe“, einmal wieder „schlafen können“. Wenn Oberlin ihn mit seinen Bekehrungsversuchen dabei stört, hält er ihm entgegen: „Wär ich allmächtig“ und „könnte das Leiden nicht ertragen, ich würde retten, retten.“ (BÜCHNER 1992:248f.) Als er über die Diskrepanz zwischen seiner Großmannssucht und der ungerührten Natur laut lachen muss: der Himmel „ein dummes blaues Aug“, der Mond darin „ganz lächerlich“, „einfältig“, da greift „der Atheismus“ in ihn und fasst ihn „ganz sicher und ruhig und fest“ (BÜCHNER:1992:242). Schließlich ist es „,der Wahnsinn“, der ihn „,packt“, ein „,unrettbarer Wahnsinn“, ein „Wahnsinn durch die Ewigkeit“ (BÜCHNER 1992:248). ${ }^{36}$ In der vielleicht tröstenden, jedenfalls aber verabschiedenden Formel ,-- - So lebte er hin“ kann beides mitgemeint sein, Atheismus und Wahnsinn. Keins von beiden steckt aber zwingend darin. Selten ist in dem weiten Horizont von Büchners Sätzen etwas strikt und nur dieses gemeint.

Woyzeck ist wieder ein anderer „Casus“. Und zwar das intendierte Stück ebenso wie die titelgebende Figur. Woyzeck als Person ist Soldat oder Rekrut $^{37}$, Faktotum für alle, die stärker als er oder nur höheren Ranges sind. Er ist Ehemann (nur ohne kirchlichen Segen) seiner Frau Marie, die ihn aber kaum versteht, sich nicht mit ihm begnügt. Er ist Barbier seines Hauptmanns, Versuchskaninchen und Objekt der Verspottung für den „Doktor“ sowie dessen (anscheinend studentisches) Publikum, Philosoph von eigenen Gnaden und nach dem Hörensagen, Pfiffikus, wenn er wieder etwas durch schieres $\mathrm{Zu}$ -

36 Philologisch müsste das Verhältnis zwischen Atheismus und Wahn noch genauer bestimmt werden; die gleiche grammatische Figur macht sie nicht inhaltlich gleich.

37 In einer Friedenszeit, doch darum nicht weniger belastet und atemlos. 
sammenreimen entdeckt hat, seinem Temperament nach zugleich phlegmatisch und cholerisch, ein Othello, wenn es ihn und „die Seine“ betrifft, Mörder mit aller Ungeschicklichkeit des Ungeübten, im Ganzen ein armer Hund und darum ein geeignetes Demonstrationsobjekt für einen Menschen überhaupt, also für den Menschen. Er ist keiner, dem andere zustimmen oder nachfolgen. Schon seiner Sprechweise wegen ${ }^{38}$ taugt er nicht zum Anführer einer Partei oder Fraktion, würde auch nicht auf die Idee kommen, andere um sich zu scharen. Mit Poesie hat er wenig zu tun. Er spitzt nur manchmal die Ohren, wenn andere etwas singen oder rezitieren. Trotzdem hat der junge Autor ihn zur Zentralfigur eines Dramas von gänzlich neuem Zuschnitt gemacht, und als diese ist er inzwischen unvergesslich.

Canetti findet durch Büchners Beschäftigung mit dieser Figur und durch seine zupackende dramaturgische Gestaltung den „,vollkommensten Umsturz in der Literatur gelungen: die Entdeckung des Geringen“ “. ${ }^{39}$ Das verwundert ein wenig, da sonst oft die Erzählung Lenz als die bahnbrechende Gestaltung des einfachen, auf allen Prunk verzichtenden Menschen gilt, der jetzt von innen heraus erfasst ist, nicht von außen als Kronzeuge vorgeführt wird. Canetti teilt aber die Epochen in Büchners Werk anders ein. Lenz ist für ihn ganz und gar die Gestaltung eines Menschen auf der Flucht, dieses vordringlichen Themas Büchners, seitdem er erfahren hat, wie die großherzogliche Polizei verfährt, nämlich sucht und verfolgt, vernimmt und festhält, auch noch Entkommene im Auge behält und ihnen über die nahe Grenze hinweg zusetzt. Erst im Woyzeck habe der junge Autor, trotz der anhaltenden und ihm bewussten Verfolgung, so viel Abstand gewonnen, dass er den „geringen“ Menschen als solchen entdecken und selbst sprechen lassen kann, ohne dabei diese Qualität des Geringen anzutasten. Canetti sieht dazu „Erbarmen“ erforderlich, das aber „stumm“ bleiben müsse, damit das Geringe ,intakt" sei. Diese „Keuschheit fürs Geringe“ findet er in Büchners Drama überzeugender durchgehalten als bei irgendeinem anderen Autor bis zu seiner eigenen Zeit. (CANETTI 1975:316; zu Woyzeck insgesamt: 318-321)

Was macht einen Menschen aus? Wie beglaubigt er sich als Mensch in einer Gesellschaft, der vor lauter Honettität ${ }^{40}$ die Humanität, auf die er sich gern

38 In den 70er Jahren des vorigen Jahrhunderts hätte man sie ,restringiert“ genannt.

39 Canetti in seiner Rede zur Verleihung des Büchner-Preises 1972 (CANETTI 1975:316).

40 Hier: militärischem Rang, wissenschaftlicher Gediegenheit und Reputation, Ansprüchen der Religion, der Moral, also der ,guten` Gesellschaft usw. 
herausredet, abhanden gekommen scheint? Büchners Drama vom „armen“ Woyzeck wirkt so, als hätte er es eigens zur Beantwortung dieser Frage konzipiert und, soweit er gekommen ist, ausgeführt. Auf allen Ebenen ist hier der Plumpe, Einfältige und Geradlinige der eigentliche Mensch. Woyzeck tritt auf als das Naturwesen, dem es um sein Menschsein geht und das danach fragt. Die Kameraden fragen kaum, schon gar nicht danach. Die überlegenen Figuren, außer Hauptmann und Doktor auch der Tambourmajor, sind so erfüllt von der Eigenart ihrer Stellung, ihres persönlichen Verdienstes, so hingegeben an die makellose Darstellung ihrer Spezialität, dass sie das permanent dienstbare Faktotum Woyzeck bestenfalls als Mitspieler oder Kontrahenten für ihre Selbstverwirklichung heranziehen. Sie kommen nicht auf die Idee, etwa nach seinen Menschenrechten oder gar seinem Recht, Mensch zu sein, zu fragen. So ist nicht nur die Anlage des Stückes, sondern auch seine Ausführung in jeder erhaltenen Szene geprägt von dem Gegeneinander von stolzen oder auch subalternen, jedenfalls selbstgewissen, im Fall des Doktors regelrecht um sich kreisenden Individuen ${ }^{41}$ und dem einen mit gehäufter Mühsal Beladenen. Nur für ihn ist sein Leben, sind seine Beziehungen zu anderen, seine Rechtfertigung vor wem auch immer ein pausenloses Problem. Wenn er auf seine Art ,philosophiert“: über das Verhalten Gottes zu den „,armen“ Kindern, die er doch auch geschaffen habe ${ }^{42}$, über das Geschick der „,armen Leut“, „,unselig“ zu sein „,in der und der andern Welt“ (wenn sie denn in den Himmel kämen, müssten sie dort „donnern helfen“; BÜCHNER 1992:155f.), dann zerlegt sich die Szene, so dialogisch auch die Repliken aufeinander eingehen mögen, in zwei Parteien, die notwendig aneinander vorbeireden. Der schändliche „Aristocratismus“ ist hier nicht mehr wie in dem Brief drei Jahre zuvor eine persönliche Untugend: Gedankenlosigkeit, Selbstsicherheit, Ichkonzentriertheit oder dergleichen. Er ist jetzt ein aufdringliches Kennzeichen der zeitgenössischen Gesellschaft, in der die Frage nach dem Selbstsein des Menschen ebenso ungehörig ist wie die nach der Verteilung der Güter und der Befugnisse. Woyzeck ist zwar als Typ überhaupt kein Revolutionär. In Büchners „Gesellschaft der Menschenrechte“ würde er nur stören, selbst wenn er nie den Mund aufmachen würde. Aber was er durch seine bloße Existenz, durch den Kontrast mit den gebietenden Herrschaften in seiner wahrscheinlich unbedeutenden Garnison vertritt, sei es

41 Die ,höheren` drei sehr auf ihre Eigenart bedacht, die anderen nicht mal daran interessiert.

42 In Poschmanns Kombinierter Werkfassung in seiner Gesamtausgabe von Büchners Werken; BÜCHNER 1992:155. 
In aller Büchner'schen Rauigkeit: Achtung ist ein Menschenrecht

stumm ausführend oder auch manchmal (ungeschickt) beredt, das gehört zum Grundstock der aufbewahrten, als Vorrat angelegten Revolution, die diese verstockte Gesellschaftsformation nötig hat. Auch seine frappierenden Äußerungen, so wenig sie zum gerade laufenden Dialog beitragen, bilden den Vorschein eines menschlicheren Umgangs miteinander. Eigentlich, über den historischen Augenblick hinaus betrachtet, kann nicht einmal die großherzogliche Residenz in den Nachwehen der ,Demagogenverfolgung ' einen solchen Umgang, zunächst also die Reflexion darüber, entbehren.

Als dramatische Figur steht Woyzeck allein da, mitunter grausam allein. Beim Militär hat er nicht nur die drei gewichtigen, reputierlichen Chargen gegen sich, sondern in peinlichen Situationen auch die „Kameraden““ ${ }^{43}$ Der treue Kamerad Andres fehlt in seiner Konfrontation mit dem Doktor wie mit dem Hauptmann. Beim Sinnieren, beim Spekulieren will ihm keiner helfen. Als er buchstäblich Blut an den Fingern hat, wird er vollends zum Outcast; auch der nur angedeutete Apparat der Justiz steht jetzt gegen ihn. Deshalb hat Büchner einiges veranstaltet, um wenigstens ,seine Seite“ zu stärken. Seiner sozialen Position nach steht Woyzeck für einen von vielen. Das „Wir“ der „,armen Leut“" verbindet ihn mit seiner kleinen Familie, mit der erzählenden Großmutter, mit dem „Idioten“ Karl, wenn man so will, mit den beiden Handwerksburschen. Sie treten nur nie zu einem Chor zusammen, es bleiben lauter vereinzelte Relationen. Damit hat Büchner den historischen Stand der sozialen Konfrontation im Großherzogtum, bevor von irgendeiner Organisation die Rede sein konnte, fatal bezeichnet (und über seine eigenen Organisationsversuche nachträglich den Stab gebrochen).

Was geht uns das heute noch an? Lässt man sich mal, selten genug, auf Büchners Werke ein - Woyzeck ist unter nur eines, unvollendet, in dieser Frage aber das radikalste -, so kann man einigen Argwohn auch gegen die heutige, vertraute Gesellschaftsordnung bekommen. Wie in den ,guten Familien' von heute die Nachfolgerin des damaligen Stiefelknechts behandelt wird, das bezeugt in der Tat eine gelungene Emanzipation gegenüber den Zuständen im frühen 19. Jahrhundert und im Großherzogtum HessenDarmstadt. ${ }^{44}$ Aber wie verhalten wir uns zu hier lebenden Menschen, die einen solchen Bezug zur Familie eingebüßt haben wie etwa die in Heime verbrachten psychisch Kranken? Wie zu denen, die einen solchen Bezug nie

43 Sie heißen selbst dann so, wenn sie es gar nicht sind.

44 Und zwar unabhängig davon, ob sie noch als ,Putzhilfe‘ oder schon als ,Raumpflegerin' oder mit noch kühneren, ätherischen Bezeichnungen tituliert wird. 
erlangt haben, Bootsflüchtlingen oder anderen ,lästigen“, weil anspruchsvollen Migranten? Zu verschämten Arbeitslosen, zu ,prekär' Beschäftigten und Bezahlten, zu Menschen guten Willens und selbstbewusster Pläne, denen ,unsere Gesellschaft' an der Leine ihrer Wirtschaftsordnung keinen angemessenen Platz bietet? Nach der Statistik machen sie heute mehrere Millionen aus. Ist es die Folge der Globalisierung, die Zusammenfassung von Individuen $\mathrm{zu}$ solchen einschüchternden, entmutigenden gewaltigen Mengen, die es so schwer bis unmöglich macht, dass wir uns zu solchen Menschen einfach als Menschen verhalten? Dass wir ihre Fähigkeiten, ihre Ideen, ihr Selbstbewusstsein ,honorieren“, indem wir auf sie achten, ihnen Raum lassen, sich zu verwirklichen? Oder diesen Raum überhaupt erst schaffen, am besten mit ihnen zusammen? Wirkt der zeitliche Abstand von den Zuständen im Großherzogtum vielleicht gar nicht, wie die Fortschrittsgläubigen voraussetzen, eo ipso zu unserem Vorteil? Ist er mit Verlusten erkauft, vielleicht gerade mit dem Verlust oder der Schwächung desjenigen Moments, von dem aus Büchner seinerzeit seine Verpflichtung zur Anteilnahme entwickelt hat? Jedenfalls übt jede geglückte Aufführung des Woyzeck einen Sog zu einer gründlich anderen Gesellschaft aus, einer Gesellschaft, in der Rücksicht auf andere Menschen ein tragendes Element wäre.

\section{Literatur}

Borgards, Roland / NeUMEYER, Harald (eds.) (21991): Büchner-Handbuch. Leben Werk - Wirkung. Stuttgart u. a.

BüCHNER, GeORg (1992 / 1994): Sämtliche Werke, Briefe und Dokumente in 2 Bänden. Hrsg. von Henri Poschmann. Frankfurt (M.).

- (1994): Briefwechsel. Kritische Studienausgabe. Hrsg. von Jan-Christoph Hauschild. Frankfurt (M.).

Büchner-Preis-Reden 1951-1971 (1981). Stuttgart.

Büchner-Preis-Reden 1972-1983 (1984). Stuttgart.

Canetti, Elias (1975): Das Gewissen der Worte. München/Wien.

Claudius, Matthias ( $\left.{ }^{2} 1991\right):$ Sämtliche Werke. München.

DEUCHERT, NORBERT (1983): Vom Hambacher Fest zur badischen Revolution: politische Presse und Anfänge deutscher Demokratie 1832-1848/49. Stuttgart.

GraB, WALTER (1989): Die französische Revolution. Aufbruch in die moderne Demokratie. Stuttgart.

HeIne, HeInRICH (1993): Sämtliche Gedichte in zeitlicher Folge. Hrsg. von Klaus Briegleb. Frankfurt (M.)/Leipzig. 
In aller Büchner'schen Rauigkeit: Achtung ist ein Menschenrecht

JANTKE, CARL (1965): Die Eigentumslosen: der deutsche Pauperismus in Darstellungen und Deutungen der zeitgenössischen Literatur. Freiburg.

LacotTe, Daniel (1987): Danton: le tribun de la Révolution. Lausanne u. a.

MARTIN, ARIANE (2007): Georg Büchner. Stuttgart.

MAYER, HANS ( $\left.{ }^{3} 1977\right)$ : Büchner und seine Zeit. Frankfurt (M.).

Mayer, Thomas Michael (1988): Georg Büchner. München (=Sonderband Text + Kritik).

Poschmann, HenRi ( $\left.{ }^{3} 1988\right)$ : Georg Büchner: Dichtung der Revolution und Revolution der Dichtung. Berlin (Ost).

SAUTERMEISTER, GeRT / SCHMID, UlRICH (eds.) (1998): Zwischen Restauration und Revolution 1815-1848. München/Wien (=Hansers Sozialgeschichte der deutschen Literatur. Bd. 5).

VolKmann, HeInRICH (1984): Sozialer Protest: Studien zu traditioneller Resistenz und kollektiver Gewalt vom Vormärz bis zur Reichsgründung. Opladen.

WeNDER, HeRBERT (1988): Georg Büchners Bild der Großen Revolution: zu den Quellen von Dantons Tod. Frankfurt (M.). 